\title{
Crack paths in composite materials
}

\author{
M. Patrício* \\ R. M. M. Mattheij* \\ *Adress: Department of Mathematics and Computer Science \\ Technische Universiteit Eindhoven
}

\begin{abstract}
Composites are often exposed to harsh loading conditions. This may lead to crack formation and propagation. In this paper an algorithm is described to predict the path of pre-existing cracks in homogeneous materials, based on an incremental approach. The approach is shown to also deal with the highly heterogeneous behaviour of periodically distributed composites.
\end{abstract}

Keywords: Composite materials, fracture, stress intensity factor, microstructure.

\section{Introduction}

Ever since Da Vinci and Galileo scientists have sought to understand why seemingly safe engineering structures sometimes fail, cf. [3, 7, 13, 14, 20, 30, 31].

For loaded linear elastic thin plate-like structures determining whether and how a given crack may propagate can be described in terms of the stress intensity factors (SIFs), cf. [2, 33]. These are material parameters that characterise the stress and displacement fields around the crack tip. It can be assumed that the crack will propagate when the circumferential SIF reaches a critical value. This behaviour can be modelled by the incremental approach described in $[11,15,18,24]$. The path that the crack will follow is predicted step by step, so that for each step the crack is incremented by updating the geometry of the problem.

Crack propagation in composite materials is still an interesting and important area of research. Composites consist of two or more chemically or physically different constituents that are bonded together along interior material interfaces and do not dissolve or blend into each other, cf. $[16,17]$. They are heterogeneous 
at the microscale, by which we mean the scale of the constituents, but they can be considered homogeneous at the macroscale. Composites are widely used in high performance products, such as aerospace components or storage tanks. This is because they need to be lightweight, yet strong enough to take harsh loading conditions.

There is great interest in predicting the behaviour of cracks on composite materials, which is complicated due to their heterogeneous nature. A major issue that prevents us from extending the incremental approach to deal with heterogeneous materials in a direct form is that determining the SIFs requires tackling the underlying elasticity problems. This is quite complex due to their highly oscillating coefficients. One other complication is due to the existence of internal material interfaces. When the crack is propagating inside one of the material components, the incremental approach for homogeneous materials can be used effectively provided we can solve the related elasticity problems. However, the crack will eventually propagate on further to other materials and interact with internal boundaries. Although we have a macro-scale problem, it is necessary to look at factors such as the nature and distribution of the constituents or the effect of the interfaces, cf. [4]. This results in an extremely complex problem which looks overwhelming when one also likes to include other aspects such as the existence of defects, the possibility of branching, creation of new cracks or delamination, cf. $[1,8,22]$.

To provide a concrete framework that captures the fundamental aspects of the problem of fracture on a composite we will consider a crack propagating through a periodically layered material. The aforementioned incremental approach will be adapted and extended for this problem by accounting for the interaction with the interfaces and making use of appropriate techniques to solve the complex elasticity problems.

In Section 2 we first introduce the equations that describe the brittle growth of a pre-existent crack present on a homogeneous isotropic linear elastic plate. The incremental approach for homogeneous materials is presented in the form of an algorithm. It is then extended to deal with highly heterogeneous periodically distributed composites, which leads to a second algorithm.

Numerical results obtained by applying the two algorithms are discussed in Section 3.

\section{Path prediction algorithm}

The phenomenon of fracture may be modelled in many different ways, depending on the type of materials, the mechanisms leading to failure or the length-scales being considered. Within the scope of linear elastic fracture mechanics, the basic equations that describe the static mechanical behaviour of a thin plate in a plane stress situation can be described by the following boundary value 
problem

$$
\begin{cases}-\nabla \cdot(\mathbf{A}(\mathbf{x}) \boldsymbol{\epsilon}(\mathbf{u}))=\mathbf{f}, & \mathrm{x} \in \Omega, \\ \mathbf{u}=\mathbf{0}, & \mathrm{x} \in \Gamma_{D}, \\ \boldsymbol{\sigma}(\mathbf{u}) \cdot \mathbf{n}=\varphi_{N}, & \mathrm{x} \in \Gamma_{N} .\end{cases}
$$

Here, the primary variable is the displacement vector $\mathbf{u}=\left(u_{i}\right)_{i=1,2}$. The stress and strain tensors denoted respectively by $\boldsymbol{\sigma}=\left(\sigma_{i j}\right)_{i, j=1,2}$ and $\boldsymbol{\epsilon}=\left(\epsilon_{i j}\right)_{i, j=1,2}$ are related by the elasticity tensor $\mathbf{A}=\left(a_{i j k h}\right)_{i, j, k, h=1,2}$. Due to the symmetry of the stress and strain tensors, the components of $\mathbf{A}$ satisfy

$$
a_{i j k h}=a_{j i k h}=a_{k h i j}, \quad \text { for } i, j, k, h=1,2,3 .
$$

In particular, for isotropic materials we have

$$
\begin{aligned}
a_{2222}=a_{1111}=\frac{E}{1-\nu^{2}} ; & a_{2211}=\frac{E \nu}{1-\nu^{2}} ; \\
a_{2121}=\frac{E}{2(1+\nu)} ; & a_{2111}=a_{2221}=0,
\end{aligned}
$$

where the Young's modulus $E$ and the Poisson's ratio $\nu$ are the relevant material parameters. For orthotropic materials the components of $\mathbf{A}$ are given in terms of the Young's moduli $E_{x y}, E_{y x}$, the Poisson's ratios $\nu_{x y}, \nu_{y x}$ and the shear modulus $G_{x y}$, so that

$$
\begin{aligned}
a_{1111} & =\frac{E_{x}}{1-\nu_{x y} \nu_{y x}} ; & a_{2211} & =\frac{E_{x} \nu_{y x}}{1-\nu_{x y} \nu_{y x}}, \\
a_{2222} & =\frac{E_{y}}{1-\nu_{x y} \nu_{y x}} ; & a_{2121} & =G_{x y}, \\
a_{2111} & =a_{2221}=0, & &
\end{aligned}
$$

where $\nu_{x y} E_{y}=\nu_{y x} E_{x}$.

When modelling brittle fracture, a crack in a linear elastic material can be considered to be a geometrical feature. The problem stated in (2.1) still holds, the crack walls being regarded as parts of the boundary of the geometry that are free of stress. Besides this boundary value problem, criteria to predict the further propagation of the crack must also be introduced. Before we can do that it is important to characterise the local stress field satisfying (2.1) in the vicinity of the crack tip. This has been determined up to an error of order of $\sqrt{r}$ for each component and is given by

$$
\boldsymbol{\sigma}(r, \theta)=\frac{K_{I}}{\sqrt{2 \pi r}} \mathbf{f}^{I}(\theta)+\frac{K_{I I}}{\sqrt{2 \pi r}} \mathbf{f}^{I I}(\theta),
$$




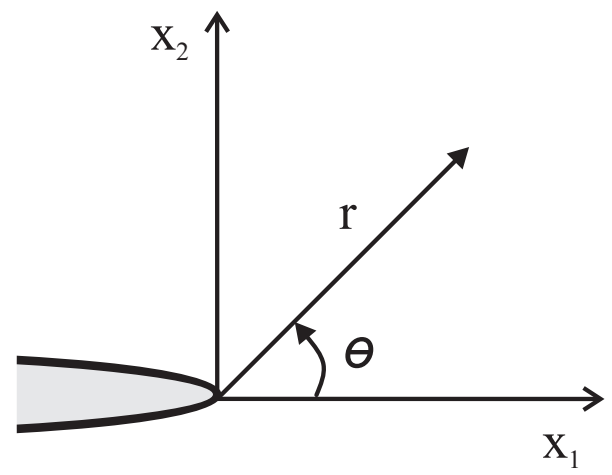

Figure 1: Crack tip region: local coordinate system.

cf. for example $[2,5,12]$. Here the polar coordinates $(r, \theta)$ are with respect to the local coordinate system represented in Figure 1. This is centred at the crack tip such that the crack is positioned along the negative horizontal semiaxis. Moreover, in (2.7) the angular variation functions $\mathbf{f}^{I}=\left(f_{i j}^{I}\right)_{i, j=1,2}$ and $\mathbf{f}^{I I}=\left(f_{i j}^{I I}\right)_{i, j=1,2}$ can be found for example in [32], whereas the SIFs are given by

$$
\begin{aligned}
K_{I} & :=\lim _{r \rightarrow 0} K_{I}^{*}(r), \text { where } K_{I}^{*}(r)=\sqrt{2 \pi r} \sigma_{22}(r, 0), \\
K_{I I} & :=\lim _{r \rightarrow 0} K_{I I}^{*}(r), \text { where } K_{I I}^{*}(r)=\sqrt{2 \pi r} \sigma_{12}(r, 0) .
\end{aligned}
$$

Now, we rewrite the stresses in (2.7) into polar coordinates to obtain

$$
\sigma_{\theta \theta}(r, \theta)=\frac{K_{\theta \theta}(\theta)}{\sqrt{2 \pi r}}
$$

where $\sigma_{\theta \theta}$ is the circumferential tensile stress given by

$$
\sigma_{\theta \theta}=\frac{\sigma_{11}+\sigma_{22}}{2}-\frac{\sigma_{11}-\sigma_{22}}{2} \cos (2 \theta)-\sigma_{12} \sin (2 \theta),
$$

and $K_{\theta \theta}$ is the circumferential stress intensity factor. The latter is in turn related to the SIFs by

$$
K_{\theta \theta}(\theta)=K_{I} \cos ^{3}\left(\frac{1}{2} \theta\right)-3 K_{I I} \sin \left(\frac{1}{2} \theta\right) \cos ^{2}\left(\frac{1}{2} \theta\right),
$$

cf. $[2,5]$. The maximum circumferential tensile stress criterion states that crack growth will occur when the maximum of $K_{\theta \theta}(\theta)$ reaches the critical stress intensity factor $K_{I_{c}}$, i.e.,

$$
\max _{\theta} K_{\theta \theta}(\theta)=K_{I_{c}}
$$


The direction for crack growth is defined by the angle $\theta_{p}^{(K)}$ which maximises $K_{\theta \theta}(\theta)$,

$$
\theta_{p}^{(K)}=2 \arctan \left(\frac{K_{I}-\sqrt{K_{I}^{2}+8 K_{I I}^{2}}}{4 K_{I I}}\right) .
$$

Whether a crack will propagate and in what direction it will do so can then be determined in terms of the SIFs. It is important to be able to compute these parameters accurately, see $[25,34]$ for a review of different methods. We note that to compute the SIFs for a given cracked plate the related elasticity problem must be solved as a first step. For composites this may require the adoption of adequate techniques.

\section{$2.1 \quad$ Homogeneous materials}

Consider a pre-existent crack on a brittle homogeneous linear elastic isotropic plate, under plane stress conditions. Denote the initial crack tip coordinates with respect to a fixed coordinate system by $\mathbf{x}_{t i p}^{(0)}=\left(x_{t i p}^{(0)}, y_{t i p}^{(0)}\right)$. Let the angle that the initial crack line forms with the direction of the $x_{1}$-axis be given by $\theta^{(0)}$. Our goal is to set up a procedure to predict the path that the crack will take.

Following an incremental approach to keep track of the position of the crack tip as the crack grows as suggested in $[15,18,24]$, the path is determined step by step. At each step the direction of propagation is computed and the crack is incremented with a line segment of length $\Delta a$. This procedure is structured as follows.

\section{Algorithm 1}

Set $n=0$. Given: $\Delta a, \mathbf{x}_{t i p}^{(0)}, \theta^{(0)}$.

1- Solve the elasticity problem.

2- Compute the values of the stress intensity factors, $K_{I}$ and $K_{I I}$.

3 - Determine the angle of propagation $\theta_{p}=\theta_{p}^{(K)}$ using equation (2.14).

4- Update the crack angle and the crack tip coordinates

$$
\begin{gathered}
\theta^{(n+1)}:=\theta^{(n)}+\theta_{P}, \\
\mathbf{x}_{t i p}^{(n+1)}=\mathbf{x}_{t i p}^{(n)}+\Delta a\left(\cos \left(\theta^{(n+1)}\right), \sin \left(\theta^{(n+1)}\right)\right) .
\end{gathered}
$$

5- Increment $n, n \rightarrow n+1$.

6- If required to determine the path further, repeat steps 1 to 6 . 
This algorithm can then be employed to determine the set of consecutive crack tip coordinates $\mathbf{x}_{t i p}^{(n)}=\left(x_{t i p}^{(n)}, y_{t i p}^{(n)}\right)$ for a propagating crack. The increment size $\Delta a$ should be small enough so that the predicted path would be roughly the same if we took an increment of a smaller length. An example of application of this procedure will be given in Section 3 .

\subsection{Heterogenous materials}

The previous analysis will now be extended to deal with a crack propagating on a linear elastic plate under plane stress conditions, constituted by a highly heterogeneous composite material. This implies that a growing crack will likely interact with more than one material and also with the various interfaces. At a given moment we can then distinguish between the situations when the crack tip region is contained inside one of the components of the composite and when the tip is at an interface.

Naturally, these two situations are not likely to be static. A crack that has its tip at the interface may be directed away from it or even continue propagating between the materials, i.e., delaminating. Also, any crack propagating through a material component may encounter an interface. When this happens several situations are possible. The crack may penetrate into the adjacent material, eventually undergoing a change in angle, reflect back to the material it came from or be deflected along one or both sides of the interface so that delamination occurs, see Figure 2 and cf. [10, 21, 28].

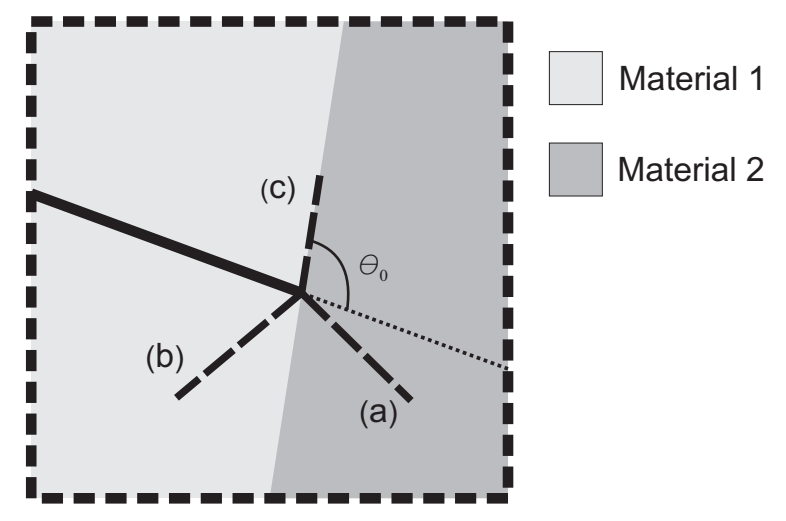

Figure 2: The crack (full line) may penetrate (a), be reflected back (b), or deviate along the interface (c).

For reasons of simplicity we will assume that the components of the composite are well bonded so that no delamination may occur. We will also disregard the possibility of branching, creation of new cracks or the existence of flaws in the 
plate as well as of interfaces between more than two materials. One may e.g. consult $[1,8,22]$ for this.

For a crack present in a linear elastic plate with isotropic homogeneous constituents, the stress field is thus characterised by (2.7), assuming the crack tip is laying inside one of the constituents. This is no longer the case when the crack tip is positioned at an interface. Let us consider the situation depicted in Figure 2 where the crack is terminating at the interface. The order of the stress singularity is then characterised by the solution $\lambda$ of the equation

$$
\begin{aligned}
& {\left[\alpha+\beta^{2}-(1-\beta)(\alpha-\beta)\left(1-\cos \left(2 \theta_{0}\right)\right) \lambda^{2}\right.} \\
& \left.+\left(1-\beta^{2}\right)\left(\pi-2 \theta_{0}\right) \cos (\lambda \pi) \cos (\lambda)\right]^{2} \\
& +\left(1-\beta^{2}\right)\left(\pi-2 \theta_{0}\right) \sin ^{2}(\lambda)\left[\left(1-\beta^{2}\right) \cos ^{2}(\lambda \pi)+\beta^{2}-\alpha^{2}\right]=0
\end{aligned}
$$

where $\alpha$ and $\beta$ are the so-called Dundurs bimaterial parameters, cf. [6, 9, 21]. Let us denote by $\sigma_{\theta \theta}^{m}$ and $\sigma_{r \theta}^{m}$ the tangential and radial stress fields defined over the material $m, m=1,2$, where the axes are positioned as represented in Figure 1. Then when $\lambda$ is a real number these fields satisfy

$$
\sigma_{\theta \theta}^{m}+i \sigma_{r \theta}^{m}=r^{\lambda-1} \mathbf{F}^{m}\left(\theta, \alpha, \beta, K_{I}, K_{I I}\right) .
$$

When $\lambda=\operatorname{Re}(\lambda)+i \operatorname{Im}(\lambda)$ is complex, they read

$$
\sigma_{\theta \theta}^{m}+i \sigma_{r \theta}^{m}=r^{\operatorname{Re}(\lambda)-1} \mathbf{G}^{m}\left(\theta, \alpha, \beta, K_{I}, K_{I I}\right) .
$$

The complex functions $\mathbf{F}^{m}$ and $\mathbf{G}^{m}$ can be found for example in [4], where the SIFs $K_{I}$ and $K_{I I}$ are also defined.

We are now ready to predict the propagation of a crack in a composite material. When the crack growth occurs far from any interface, the incremental approach of Algorithm 1 is applicable. This implies solving an elasticity problem at each iteration using numerical methods, finding approximations for the SIFs and computing the propagation angle from these by using the maximum circumferential tensile stress criterion. The crack is then incremented, i.e. the geometry is updated by subtracting the portion of new crack created.

When the crack tip is at the interface, the stress fields display the more complex behaviour described by (2.18) or (2.19). We assume that the maximum circumferential tensile stress criterion can again be used to predict the direction of propagation. We approximate this direction by averaging the amplitudes of a number of angles, each maximising $\sigma_{\theta \theta}$ on a small circle centred at the crack tip. The stress fields are computed by the finite element method.

It is important to note that in the region near an interface the accuracy of the computed stress fields is less than elsewhere, cf. [29]. To deal with this, 
our strategy is to adjust the size of the new crack increment when the crack tip approaches this critical region. The propagation angle is kept unchanged and the updated crack tip position lays at the interface. This seems to be a good idea for situations when the angle $\theta_{0}$ represented in Figure 2 is not expected to be close to $0^{\circ}$ or $180^{\circ}$. If the crack may grow nearly parallel to the interface line, a different approach should be used. Not only small errors in the propagation angle may lead to very different updated crack tip positions, but also cracks parallel to layers behave differently from those that propagate through the layers, see for example [29] and references within.

The procedures we have described can be structured in the following algorithm for a composite plate with a pre-existent crack. We denote by $\Delta a$ and $\delta_{R}$ the length for the crack increments and the minimum distance between the crack tip and the interface for which we assume the stress fields are accurately computed. Moreover, let $\mathbf{x}_{t i p}^{(n)}$ denote the crack tip coordinates with respect to a fixed coordinate system at the iteration step $n$. Finally, $\theta^{(n)}$ is the angle between the crack segment containing the crack tip and the direction of the $x_{1}$-axis of the coordinate system.

\section{Algorithm 2}

Set $n=0$. Given: $\Delta a, \delta_{R}, \mathbf{x}_{t i p}^{(0)}, \theta^{(0)}$.

1- Solve the elasticity problem.

2- If $\mathbf{x}_{t i p}^{(n)}$ is at an interface, compute $\sigma_{\theta \theta}$ using (2.11). Otherwise skip to step 4. 3 - Compute the value of the propagation angle $\theta_{P}$ using the maximum circumferential tensile stress criterion. Skip to step 6 .

4- Compute the values of the stress intensity factors $K_{I}$ and $K_{I I}$.

5- Determine the angle of propagation $\theta_{P}=\theta_{P}^{(K)}$ using equation (2.14).

6- Update the crack angle and the crack tip coordinates

$$
\begin{gathered}
\theta^{(n+1)}:=\theta^{(n)}+\theta_{P}, \\
\mathbf{x}_{t i p}^{(n+1)}:=\mathbf{x}_{t i p}^{(n)}+\Delta a\left(\cos \left(\theta^{(n+1)}\right), \sin \left(\theta^{(n+1)}\right)\right) .
\end{gathered}
$$

7- Find the coordinates $\mathbf{y}^{(n+1)}$ of the point in the interface that is collinear with the points of coordinates $\mathbf{x}_{t i p}^{(n)}$ and $\mathbf{x}_{t i p}^{(n+1)}$. If $\left\|\mathbf{y}^{(n+1)}-\mathbf{x}_{t i p}^{(n+1)}\right\|<\delta_{R}$ replace $\mathbf{x}_{\text {tip }}^{(n+1)}$ by $\mathbf{y}^{(n+1)}$,

$$
\mathbf{x}_{t i p}^{(n+1)} \rightarrow \mathbf{y}^{(n+1)}
$$

8- Increment $n, n \rightarrow n+1$.

9- If required to determine the path further, repeat steps 1 to 9 . 
The approach expressed in this algorithm can be extended to include the aspects that have been precluded such as the possibility of branching or delamination. However, the added complexity will translate into the necessity of much more work.

\section{$3 \quad$ Numerical results}

The previous section was devoted to the analysis of the phenomenon of crack growth on linear elastic plates. Procedures based on the existing incremental approach were set up as algorithms for homogeneous materials and for composites. In what follows we will use the previous algorithms to predict the paths of cracks for two different problems.

\subsection{Homogeneous plate}

Consider the cracked rectangular linear elastic homogeneous plate of width $b$ and height $2 b$ represented in Figure 3. Let the ratio between the length of the crack measured along the $x_{1}$-axis and the width of the plate be such that $a / b=1 / 5$. In order to consider a mixed mode situation the inclined initial crack is considered to be at a $\alpha=45^{\circ}$ angle with the horizontal axis. Without loss of generality, we take $b=1$.

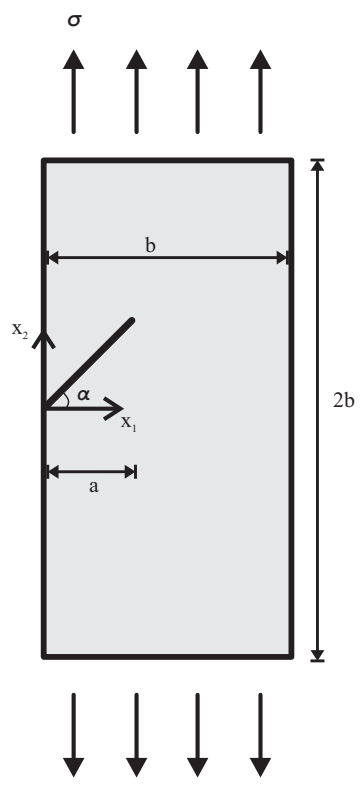

Figure 3: Finite plate with a crack subject to mixed mode loading.

If the uniform tensile stress $\sigma$, which is applied at the lower and upper hor- 
izontal boundaries of the plate, is large enough so that the fracture criterion (2.13) holds, we do know that the initial crack will propagate. By employing Algorithm 1, the set of consecutive crack tip coordinates $\mathbf{x}_{t i p}^{(n)}=\left(x_{t i p}^{(n)}, y_{t i p}^{(n)}\right)$ for the propagating crack can be determined.

We start by taking $\Delta a=0.025$. The numerical package Abaqus is employed to solve the elasticity problem in the first step of the algorithm. Quadratic rectangular elements are employed everywhere except near the crack tip, where quadratic triangular elements are used. The SIFs are determined using the Jintegral method, cf. [25, 34]. The results obtained using the aforementioned algorithm are displayed in Table 1.

\begin{tabular}{|c|c|c|}
\hline $\mathrm{n}$ & $x_{\text {tip }}^{(n)}$ & $y_{\text {tip }}^{(n)}$ \\
\hline 0 & 0.100 & 0.100 \\
\hline 1 & 0.125 & 0.102 \\
\hline 2 & 0.150 & 0.101 \\
\hline 3 & 0.175 & 0.101 \\
\hline 4 & 0.200 & 0.101 \\
\hline
\end{tabular}

\begin{tabular}{|c|c|c|}
\hline $\mathrm{n}$ & $x_{\text {tip }}^{(n)}$ & $y_{\text {tip }}^{(n)}$ \\
\hline 5 & 0.225 & 0.101 \\
\hline 6 & 0.250 & 0.101 \\
\hline 7 & 0.225 & 0.101 \\
\hline 8 & 0.300 & 0.101 \\
\hline 9 & 0.325 & 0.101 \\
\hline
\end{tabular}

\begin{tabular}{|c|c|c|}
\hline $\mathrm{n}$ & $x_{\text {tip }}^{(n)}$ & $y_{\text {tip }}^{(n)}$ \\
\hline 10 & 0.350 & 0.101 \\
\hline 11 & 0.375 & 0.101 \\
\hline 12 & 0.400 & 0.102 \\
\hline 13 & 0.425 & 0.102 \\
\hline 14 & 0.475 & 0.102 \\
\hline
\end{tabular}

Table 1: Crack tip coordinates.

The discretisation elements of the crack tip region are illustrated in Figure 4, where the crack is represented by the darker region.

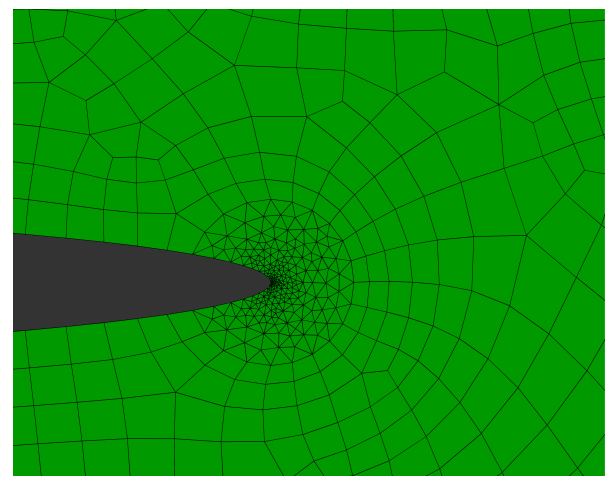

Figure 4: Crack tip region.

The deformed state of the plate before propagation and the deformed plate geometry obtained after applying 15 iterations of the algorithm are displayed in Figure 5 a) and b), respectively. 


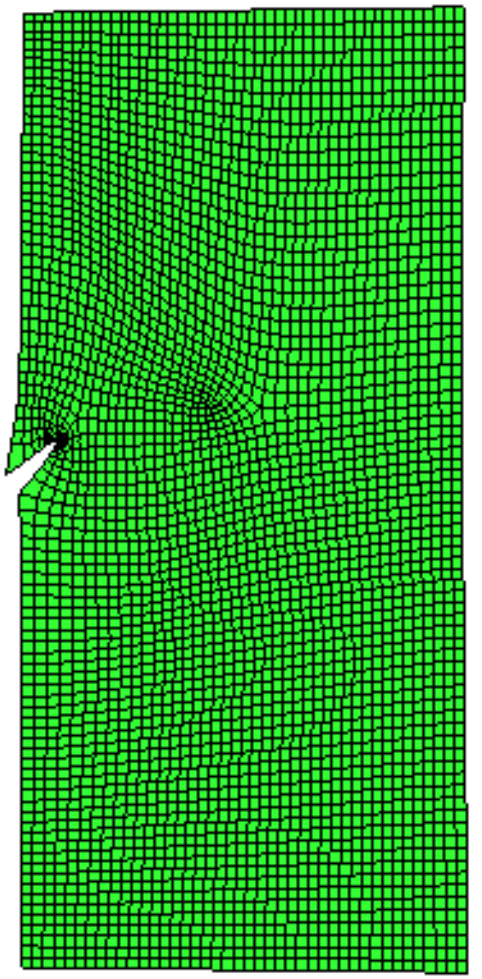

Figure 5 - a) Deformed initial plate.

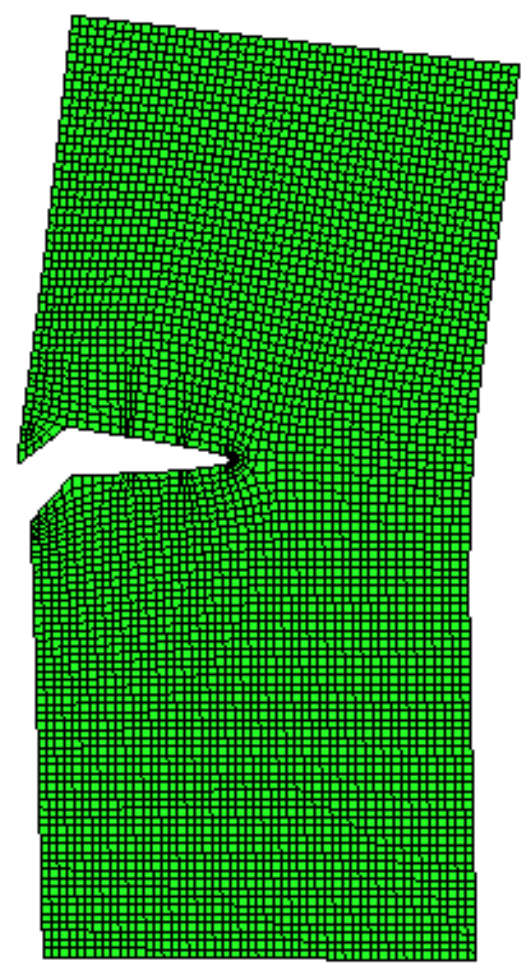

b) Deformed plate for $n=15$.

Finally, the crack path can be seen in Figure 6. In a) we plot the initial crack segment and in b) the crack path as it was determined after 15 iterations.

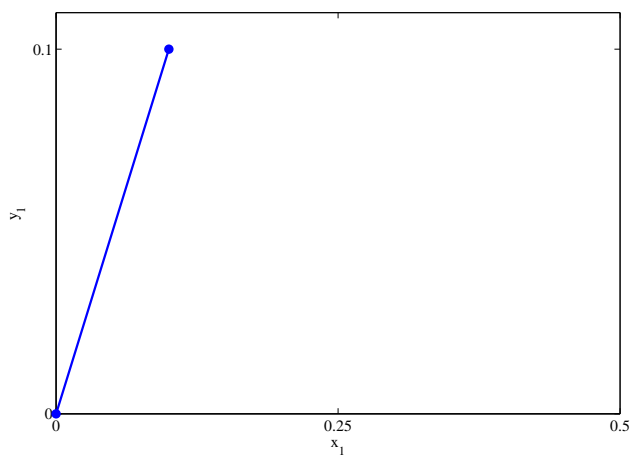

Figure 6 - a) Initial crack position.

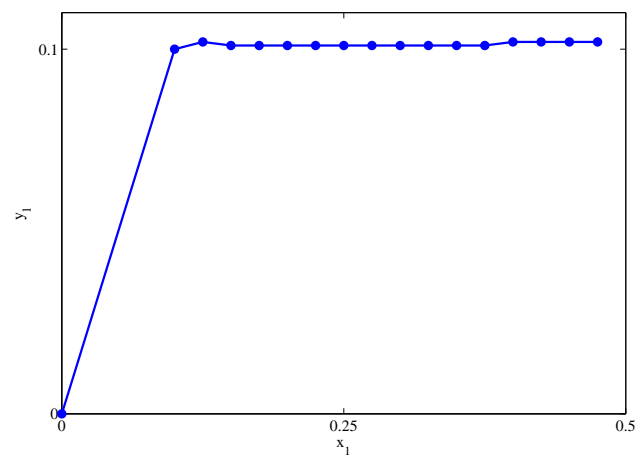

b) Crack path for $n=15$.

There appears to be a strong tendency for the crack to propagate mainly in mode $I$, during continued fracture. This agrees with the predictions of $[5,15]$. We also observe that this simulation of crack growth is based on the static stress 
intensity factors. Dynamic effects such as wave propagation are not taken into account. A dynamic analysis is presented in [32].

\subsection{Heterogeneous plate}

To illustrate the prediction of crack growth on a heterogeneous plate we will consider a periodically layered cracked plate $\Omega=([-0.5,0.5] \times[-0.5,0.5]) \backslash \Omega_{C}$, the crack line being given by $\Omega_{C}=[-0.50,-0.49] \times\{0\}$, cf. Figure 7 . The layers are at a $45^{\circ}$ angle with the horizontal axis and the two components of the plate are isotropic, homogeneous and linear elastic, being characterised by $E_{1}=1, \nu_{1}=0.1$ and $E_{2}=10, \nu_{2}=0.3$.

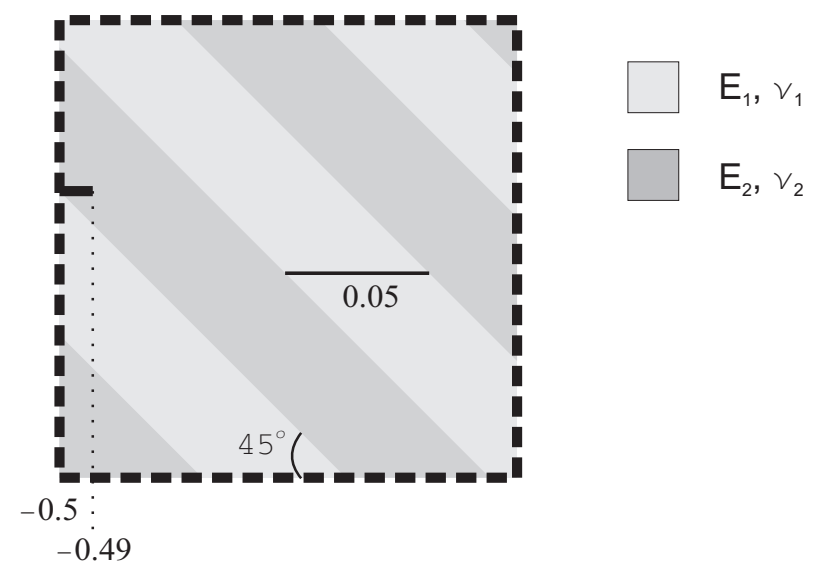

Figure 7: Zoom in of the crack tip region.

To complete the problem formulation, we consider the following boundary conditions

$$
\boldsymbol{\varphi}_{N}(\mathbf{x})= \begin{cases}(0,1), & x_{2}=0.5 \\ (0,-1), & x_{2}=-0.5 \\ (0,0), & \text { otherwise }\end{cases}
$$

and assume that body forces are absent. Our goal is to predict the path of the crack assuming that the loading is sufficiently large to ensure crack growth. We will employ Algorithm 2 with $\Delta a=0.01$ and $\delta_{R}=0.0125$ to obtain a reference solution.

For each iteration, the elasticity problem is solved by the finite element method using fine meshes, composed of quadratic triangular elements near the crack tip and quadratic rectangular elements elsewhere. The J-integral method is employed to compute the SIFs in the fourth step of the algorithm. 


\begin{tabular}{|c|c|c|c|}
\hline $\mathrm{n}$ & $(\Delta a)_{x_{1}}$ & $(\Delta a)_{x_{2}}$ & $\theta_{P}$ \\
\hline 0 & $9,89 E-3$ & $1.48 E-3$ & $8.52^{\circ}$ \\
\hline 1 & $9.95 E-3$ & $-9.90 E-4$ & $-14.20^{\circ}$ \\
\hline 2 & $9.82 E-3$ & $-1.88 E-3$ & $-5.16^{\circ}$ \\
\hline 3 & $1.82 E-2$ & $-7.09 E-3$ & $-9.81^{\circ}$ \\
\hline
\end{tabular}

\begin{tabular}{|c|c|c|c|}
\hline $\mathrm{n}$ & $(\Delta a)_{x_{1}}$ & $(\Delta a)_{x_{2}}$ & $\theta_{P}$ \\
\hline 4 & $9.85 E-3$ & $1.74 E-3$ & $30.65^{\circ}$ \\
\hline 5 & $9.99 E-3$ & $4.85 E-4$ & $-12.78^{\circ}$ \\
\hline 6 & $1.00 E-2$ & $1.13 E-4$ & $3.43^{\circ}$ \\
\hline 7 & $1.65 E-2$ & $2.29 E-3$ & 7.27 \\
\hline
\end{tabular}

Table 2: Crack tip increments.

This procedure enables us to predict the amplitude of the propagation angles $\theta_{P}$ of the new crack segments, as well as the corresponding horizontal and vertical components $(\Delta a)_{x_{1}}$ and $(\Delta a)_{x_{2}}$ of the increment vector. These values are displayed in Table 2 for the first 8 increments.

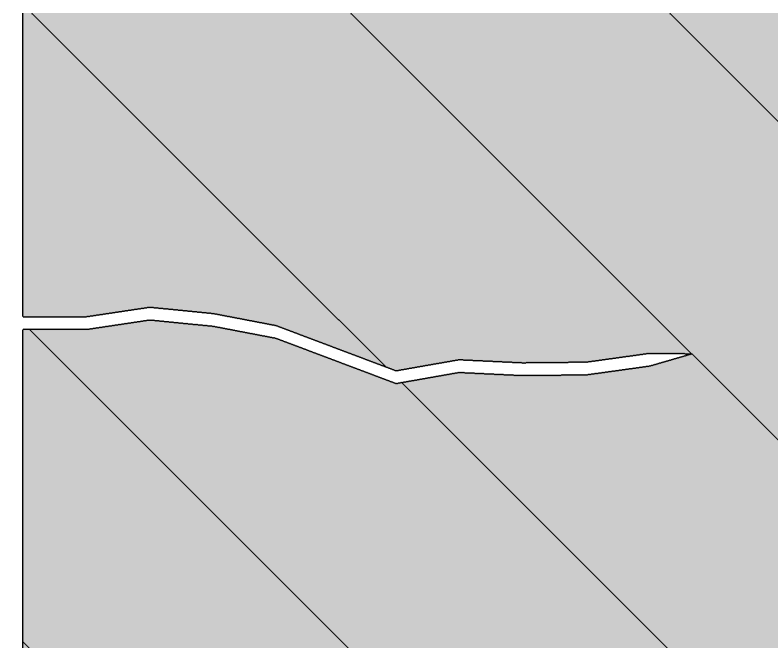

Figure 8: Crack propagating through layers.

We note that the lengths of the increments when $n=3$ and $n=7$ are larger. This is due to the proximity of the crack tip to the interface. The updated crack tip positions were computed with step 7 of the algorithm.

Since the plate is pulled in the vertical direction, the crack propagates essentially in a horizontal direction, as can be seen in Figure 8.

We also note that it is possible to solve the elasticity problems because the thickness of the constituents of the plate we have considered is not very small. For a more heterogeneous problem employing finite elements directly is not an option, as we would have to use fine meshes with a prohibitive computational cost. A second approximation for the crack path is then obtained by 
employing the hybrid approach described in [27] to solve the first step of Algorithm 2 for each iteration. The idea of this approach is to reduce the computational complexity of the problem. Since we consider one crack only, it is not necessary to employ domain decomposition in the hybrid approach. The procedure to find an approximation for the solution of each elasticity problem then consists of homogenising the material of the plate outside of the rectangle $\Omega_{1}=([-0.5,0.25] \times[-0.25,0.25]) \backslash \Omega_{C}$ that contains the crack. Next we solve the elasticity problem for a plate consisting of three distinct materials, the original material components over $\Omega_{1}$ and the homogenised material over $\Omega \backslash \Omega_{1}$. The crack path computed using Algorithm 2 with the hybrid approach is depicted by dots in Figure 9. There we also show the path obtained by using the reference solution, represented as a full line. We can see that the plots almost coincide.

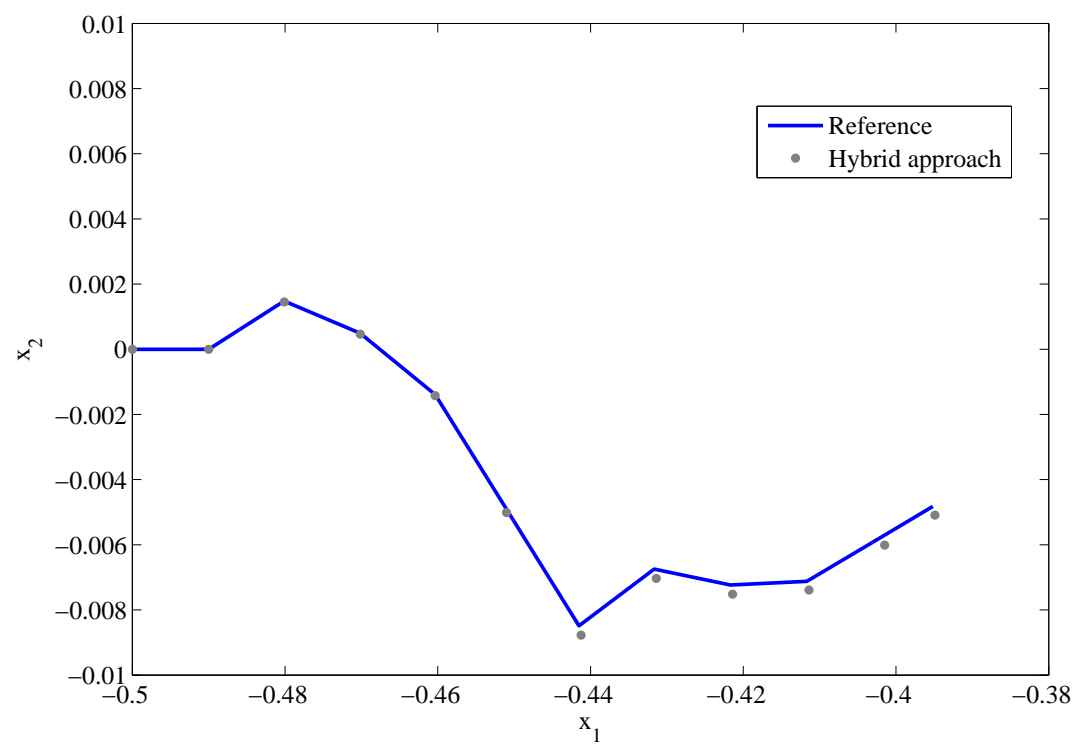

Figure 9: Paths determined by application of Algorithm 2.

It is then a good strategy to employ the hybrid approach to solve the elasticity problems when one wants to predict crack propagation for highly composite materials. This will actually work better for materials with smaller heterogeneities due to the decrease in the homogenisation error. In that case, the size of the region where we have to perform a microscopic analysis can be smaller.

Finally, we note that a macroscopic approach can be adopted to find the path for the homogenised plate, which is constituted of an orthotropic material. The 
material constants may be found in [26]. The procedure is much more simple: after employing homogenisation, one can simply apply Algorithm 1. However, since the homogenised material is orthotropic, the maximum circumferential tensile stress criterion would need to be reformulated, see for example [19, 23]. Moreover, using the homogenised model implies losing the effects of locality, as well as the influence of the internal boundaries on the path, unless the latter aspect is incorporated somehow. To have a microscopic resolution, homogenisation may not be employed in the vicinity of the crack tip.

\section{References}

[1] L. Banks-Sills and D. Ashkenazi. A note on fracture criteria for interface fracture. Int. J. Fract., 103(2):177-188, 2000.

[2] D. Broek. Elementary engineering fracture mechanics. Kluwer Academic Publishers, 1986.

[3] L. Ceriolo and A. Tommaso. Fracture mechanics of brittle materials: a historical point of view. 2nd Int. PhD Sympodium in Civil Engineering, 1998.

[4] J. Chang and J. Xu. The singular stress field and stress intensity factors of a crack terminating at a bimaterial interface. Int. J. Mech. Sci., 49, 2007.

[5] G. P. Cherepanov. Mechanics of brittle fracture. MacGraw-Hill, 1979.

[6] A. Cirello and B. Zuccarello. On the effects of a crack propagating toward the interface of a bimaterial system. Eng. Fract. Mech., 73(9):1264-1277, 2006.

[7] B. Cotterell. The past, present, and future of fracture mechanics. Eng. Fract. Mech., 69:533-553, 2002.

[8] I. Demir, H. M. Zbib, and M. Khaleel. Microscopic analysis of crack propagation for multiple cracks, inclusions and voids. Theor. Appl. Fract. Mech., 36:147-164, 2001.

[9] J. Dundurs. Discussion of edge-bonded dissimilar orthogonal elastic wedges under normal and shear loading. J. Appl. Mech., 36, 1969.

[10] F. Erdogan and T. S. Cook. Antiplane shear crack terminating at and going through a bimaterial interface. Int. J. Fract., 10:227-240, 1974.

[11] F. Erdogan and G. C. Sih. On the crack extension in plates under plane loading and transverse shear. J. Basic Eng., 85:519-527, 1963.

[12] L. B. Freund. Dynamic fracture mechanics. Cambridge University Press, 1990. 
[13] G. Galilei. Discorsi e dimonstrazioni matematiche intorno duo nuove scienze, 1638.

[14] A. A. Griffith. The phenomena of rupture and flows in solids. Phil. Trans. Roy. Soc. London, A221:163-198, 1921.

[15] D. Hegen. An element-free Galerkin method for crack propagation in brittle materials. PhD thesis, Technical University of Eindhoven, 1997.

[16] L. Mishnaevsky Jr. Computational mesomechanics of composites: numerical analysis of the effect of microstructures of composites on their strength and damage resistance. John Wiley, 2007.

[17] A. K. Kulshreshtha and C. Vasile. Handbook of polymer blends and composites. Rapra Technology Ltd., 2002.

[18] R. W. Lewis and B. Koosha. A mixed mode rock fracture model for the prediction of crack path. Int. J. Numer. Anal. Meth. Geomech., 23:281294, 1999.

[19] W. Lim, S. Choi, and B. Sankar. Biaxial load effects on crack extension in anisotropic solids. Eng. Fract. Mech., 68:403-416, 2001.

[20] J. R. Lund and J. P. Byrne. Leonardo da Vinci's tensile strength tests: Implications for the discovery of engineering mechanics. Civ. Eng. Environ. Syst., 18(3):243-250, 2001.

[21] K. Madani, M. Belhouari, B. Bachir Bouiadjra, B. Serier, and M. Benguediab. Crack deflection at an interface of alumina/metal joint: A numerical analysis. Comput. Mater. Sci., 38:625-630, 2006.

[22] M. A. Meggiolaro, A. C. O. Miranda, J. T. P. Castroa, and L. F. Martha. Stress intensity factor equations for branched crack growth. Eng. Fract. Mech., 72:2647-2671, 2005.

[23] L. Nobile and C. Carloni. Fracture analysis for orthotropic cracked plates. Compos. Struct., 68:285-293, 2005.

[24] M. Patrício and R. Mattheij. Crack propagation analysis. CASA report 07-03, 2007.

[25] M. Patrício, R. Mattheij, and G. de With. Effects of the local structure on a cracked periodically distributed composite. Submited to Comput. Model. Eng. Sci., 2008.

[26] M. Patrício, R. Mattheij, and G. de With. Homogenisation with application to layered materials. Math. Comput. Simulat., 79:288-305, 2008.

[27] M. Patrício, R. Mattheij, and G. de With. Solutions for periodically distributed materials with localised imperfections. Submited to CMES: Comput. Model. Eng. Sci., 2008. 
[28] S. Roham, K. Hardikar, and P. Woytowitz. Crack penetration and deflection at a bimaterial interface in a four-point bend test. J. Mater. Res., 19:3019-3027, 2004.

[29] M. T. Tilbrook, R. Moon, and M. Hoffman. Crack propagation in graded composites. Compos. Sci. Technol., 65:201-220, 2005.

[30] S. P. Timoshenko. History of the strength of materials. MacGraw-Hill, 1953.

[31] L. da Vinci. Codice Atlantico. Folio 82 recto-B, date unknown.

[32] J. C. W. van Vroonhoven. Dynamic crack propagation in brittle materials: analyses based on fracture and damage mechanics. $\mathrm{PhD}$ thesis, Technical University of Eindhoven, 1996.

[33] G. de With. Structure, deformation and integrity of materials. Wiley-VCH Verlag, 2006.

[34] A. Zehnder. Lecture notes on fracture mechanics. Cornell University, 2007. 